\title{
OPTIONS FOR A NEW ARIZONA OBSERVATORY: CONFIGURATIONS, COSTS AND SITE
}

\author{
N. J. Woolf and J. R. P. Angel \\ Steward Observatory, University of Arizona
}

J. T. Williams

Multiple Mirror Telescope Observatory and Smithsonian Astrophysical Observatory INTRODUCTION

This paper is concerned with the process of creating a major observatory at minimum cost. Its premises are that cost optimization starts with an appropriately modestly priced building block, a $7.5 \mathrm{~m}$ telescope. There are many possible configurations in which this building block can be used, ranging from a single telescope or a set of separate telescopes with at most electronic data addition, to an MMT in which the light of all telescopes can be phased when desired. In addition there are options for using elements in a non-redundant linear array or Michelson inteferometer. We provide the reader with a means of estimating costs of different facilities all on the same basis, and tied to the actual cost of producing the MMT.

It is also important to place the observatory on a site which maximizes the output per unit cost of the facility. We discuss the selection process which has led to Mt. Graham in Arizona as being likely to satisfy this criterion. We give brief details of our preliminary knowledge about the site.

THE TELESCOPE UNIT, AND ITS POSSIBLE WAYS OF USE

The astronomical observatory takes in money and puts out discoveries and observations. Our goal is to work within a given budget and maximize the output. This, of course is not a real option, but since observatories ultimately receive from a limited purse, it is our responsibility to explore the cost effectiveness of our designs. To the extent we have not explored them, we should expect difficulty in obtaining support. Within the framework of existing technology we can see how best to get value for money. Most observations depend only on the total collecting area of telescopes rather than in their detailed form, provided the telescopes are large enough. The collecting area of telescopes varies as $D^{2}$, while for homologous telescopes the cost varies as $D^{2.7}$. Therefore the optimum configuration is a set of telescopes, each one of which is just "large enough".

Proceedings of the IAU Colloquium No. 79: "Very Large Telescopes, their Instrumentation and Programs", Garching, April 9-12, 1984. 
Unfortunately the concept of a "large enough" telescope is a can of worms. In the infrared, a large enough telescope is one where the seeing is excellent, but always worse than the diffraction limit. Thus "large enough" is a wavelength dependent quantity. In the visible region, large enough means that the photon noise exceeds the detector noise, and thus "large enough" is a spectral resolution and detector dependent quantity. Finally, if angular resolution is desired, there appears to be no theoretical limit to the resolution attainable by interferometry or adaptive optics, except that set by the collecting area's largest dimension. For it there is no such, thing as large enough.

Despite that, there is merit in the assertion that somewhere between 5 and $10 \mathrm{~m}$ diameter a telescope is a large enough unit. Angular resolution may be obtained by combining the output of such units. Seeing at good sites would be worse than the telescope diffraction limit over most of the infrared, most of the time. Sky brightness causes photon noise to exceed detector readout noise for the most useful spectral resolutions and reasonable observing times at all wavelengths.

The most efficient use of current technology seems to be the production of telescopes of $\sim 2.4 \mathrm{~m}$ in size such as the Wyoming IR observatory (Gehrz and Hackwell 1978) and the new McGraw-Hill 2.4m reflector (Robinson 1982, Hiltner 1984) with a $\$ 1.8 \mathrm{M}$ total budget. If we could build a $7.5 \mathrm{~m}$ telescope at the same cost per unit area, it would cost only $\$ 17.5 \mathrm{M}$. If we were to scale as $D^{2.7}$, the cost would be $\$ 39 \mathrm{M}$, some 2.2 times higher. Our concern is to produce building blocks for an observatory comparable with the excellent cost per unit area ratio of these mature technology $2.4 \mathrm{~m}$ telescopes.

The McGraw-Hill cost of $\$ 0.4 \mathrm{M}$ (1984) per square meter shows how far technology has developed. The Lick 36-inch refractor and associated observatory cost $\$ 700,000$ in 1888 for $\$ 1 M(1888)$ per square meter, and the Hale Observatory produced the $5 \mathrm{~m}$ reflector at $\$ 0.3 \mathrm{M}(\sim 1935)$ per square meter. The immense inflation from those periods $(\sim 20 x)$ has been wiped out by improved efficiency of telescope production.

The design for a new observatory should recognize that, as Colin Humphries has shown at this meeting, area intensive costs such as fabrication of mirrors go as $D^{2}$, building costs tend to go as $(D F)^{3}$, and that one off items such as computers go as $D^{0}$. Thus for a new observatory, the first priority must be to bring down building size and costs. The second must be to substitute technology for brute force in the positioning of optics. Thus the pressures are to go to 
fast focal ratios, alt-azimuth drives, and servo control of optics alignment, focus, etc. In addition, it seems that in the visual region optimum value for money comes from wide field capability with parallel observations whenever possible. Although this requires more coordination of observers than currently occurs, the benefits to all of increased observing time warrant such a step.

In Table 1, we have estimated the price for various size telescope facillties on two bases. The upper line assumes equal cost per unit area. The lower assumes cost increases as $D^{2.7}$.

To the extent that performance is area independent, the top line is achievable by using an array of $2.4 \mathrm{~m}$ telescopes. It is an upper limit, since some mass production cost savings would result. This price is for a telescope

Table 1. Price in \$ (1984)

\begin{tabular}{lccccccc}
\hline Diameter (Meters) & 2.4 & 3.7 & 4.5 & 6.0 & 7.5 & 10 & 15 \\
$\begin{array}{l}\text { Equal Cost Per } \\
\text { Unit Area }\end{array}$ & $1.8 \mathrm{M}$ & $4.3 \mathrm{M}$ & $6.3 \mathrm{M}$ & $11.3 \mathrm{M}$ & $17.6 \mathrm{M}$ & $31 \mathrm{M}$ & $70 \mathrm{M}$ \\
D2.7 & $1.8 \mathrm{M}$ & $5.8 \mathrm{M}$ & $9.8 \mathrm{M}$ & $21.4 \mathrm{M}$ & $39 \mathrm{M}$ & $83 \mathrm{M}$ & $258 \mathrm{M}$ \\
Ratio & 1 & 1.35 & 1.55 & 1.90 & 2.22 & 2.7 & 3.6 \\
\hline
\end{tabular}

and bullding at an existing site, in Arizona. It does not include auxiliary instrumentation, and since the existing dormitory, etc., facilities of the McGraw Hill Observatory are adequate, the price does not include these either. None the less, the relatively similar price for the Wyoming telescope at a new site (and allowance for inflation) suggests that site development and other facilities need not increase the price by more than $\sim 30 \%$.

Large telescopes have up until now failed dismally in even reaching the lower set of numbers. One of the better ones in this respect is the $4.5 \mathrm{~m}$ effective MMT. The MMT cost inflated to today would be $\$ 15.7 \mathrm{M}$, if one assumed an (inflated) $\$ 3 M$ for the primary mirror blanks which were obtained at no cost.

The MMT costs were high because the many novel features required a higher than usual fraction of the money to go into design. None the less, it has well documented costs, and we can use it as a basis for projecting the costs of a High Techology Observatory. 
Table 2. MMT Costs Inflated to 1984

$\begin{array}{lr}\text { Building and Site Preparation } & 2.7 \mathrm{M} \\ \text { Interior Finishing } & \frac{1.5 \mathrm{M}}{4.2 \mathrm{M}} \\ \text { SUBTOTAL } & 3.0 \mathrm{M} \\ \text { Optics Blanks } & \frac{3.4 \mathrm{M}}{6.4 \mathrm{M}} \\ \quad \text { Other Optical Work } & 5.1 \mathrm{M} \\ \text { SUBTOTAL } & \underline{5.7 \mathrm{M}} \\ \text { Telescope Structure and Controls } & 15.0 \\ \text { TOTAL } & \end{array}$

These costs include design, installation, contingency and project management, but omit auxiliary instrumentation and dormitory.

The most accurate cost estimate we can make are for a telescope of the same size and mass as the MMT that will fit into a building and yoke of the same size as the MMT. Such a telescope, is shown in Figure 1 , has a $7.5 \mathrm{~m} F / 1$ primary, with the F/4 Cassegrain corrector of Epps, Angel and Anderson (1984) that yields a flat field of view of 40 arcminutes. (An even wider field $F / 1$ to $F / 2$ optical system has been described by Angel, Woolf and Epps (1982), but it suffers the disadvantages of a trapped focus and curved focal plane.) A small Cassegrain secondary with an F/1 primary would permit infrared observations. A Nasmyth flat may be inserted through the central hole of the primary, and used in conjunction with the $\sim F / 30$ IR secondary.

F/1 optics do require technical innovation in figuring. A mirror casting furnace is being set up so that $F / 1,8 \mathrm{~m}$ honeycomb paraboloids can be spun cast. At the Optical Sciences Center the University of Arizona has installed a large optical generator capable of grinding $F / 1$ paraboloids to high precision, and Angel (1984) is testing an actively controlled warped lap to permit rapid figuring. An appropriately sized test tower already exists. While we have high hopes that $F / 1$ primaries will be possible, there must remain an element of uncertainty until the method is proven. We shall therefore also consider a telescope with an $F / 1.8$ primary that could be produced by existing methods and has the advantage of yielding a $1^{\circ}$ field. The impact on cost is not we believe on the mirror itself. If the new method works, $F / 1$ and $F / 1.8$ costs will not differ significantly. The difference is mostly in the enclosure cost.

Let us now consider the cost of these units. The mirror casting program is committed to producing a $3.5 \mathrm{~m}$ blank. Beyond this, the estimated cost of 

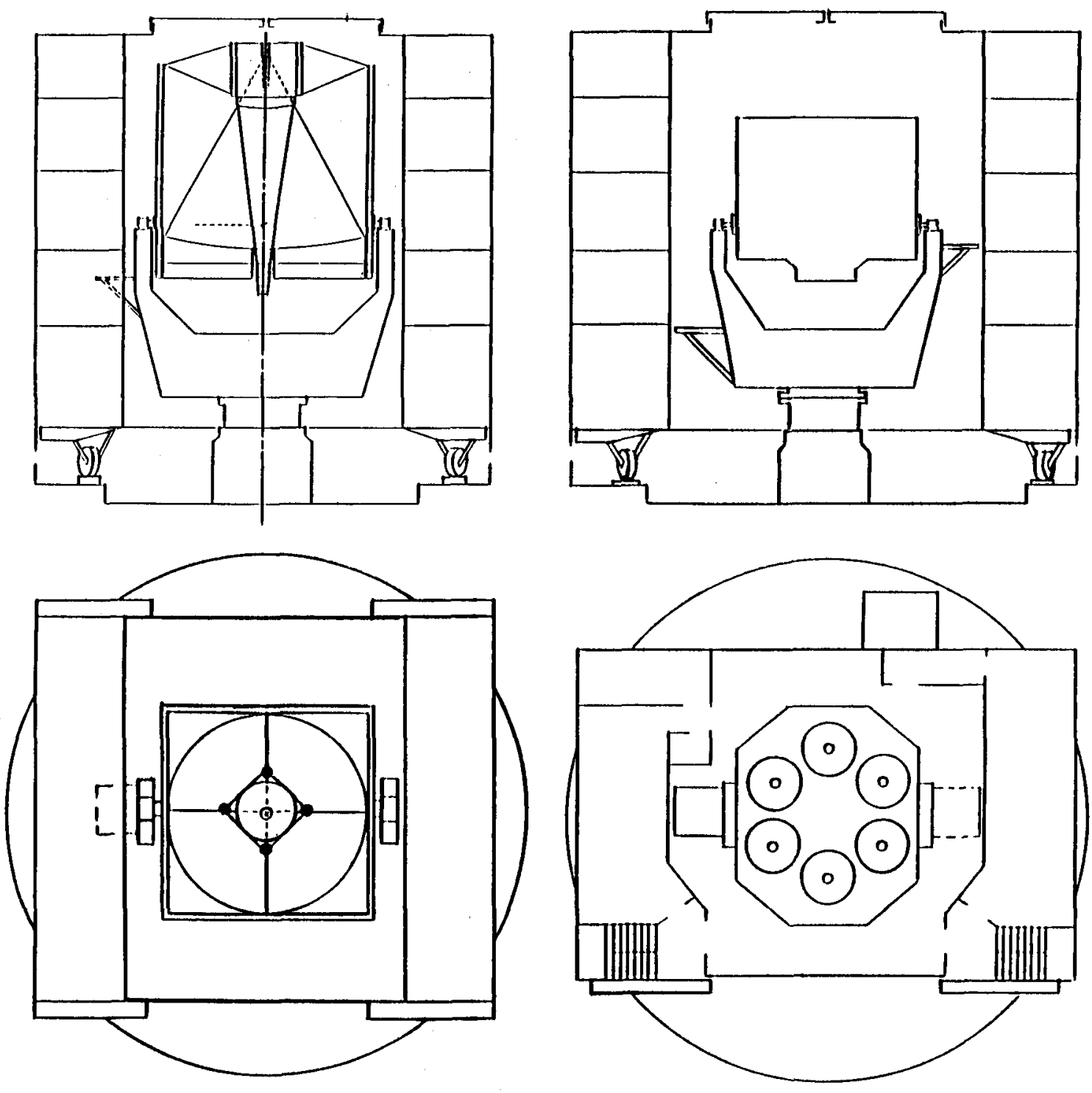

$7.5 \mathrm{~m}$

MMT 
producing a first $7.5 \mathrm{~m}$ blank is estimated as $\$ 2.5 \mathrm{M}$. Further blanks are likely to cost about $\$ 1.5 \mathrm{M}$ each. Estimates of fabricating a finished mirror suggest that a further $\$ 3.5 \mathrm{M}$ would be required for the first one. We may estimate total production costs for primary optics as $\$ 6 \mathrm{M}$ for the first, and $\$ 4 \mathrm{M}$ averaged over a set of further blanks. Infrared secondaries would be a minor item, but wide field optics with a $1.5 \mathrm{~m}$ secondary, three corrector lenses, and atmospheric dispersion correcting prisms would not. We estimate $\$ 0.3 \mathrm{M}$ for optical blanks and $\$ 1.5 \mathrm{M}$ for figuring, with the fabrication cost decreasing to $\$ 1 \mathrm{M}$ for successive units. The much larger correctors and secondary for F/1.8 primaries would be more expensive.

In Table 3 we have generated a table so that costs may be estimated.

Table 3.

\begin{tabular}{lrc}
\hline & Cost of First Item & Further Per Item Cost \\
Primary Mirror (F/1 or F/1.8) & $\$ 6.0 \mathrm{M}$ & $\$ 4.0 \mathrm{M}$ \\
IR Secondary and Chopping System & $0.4 \mathrm{M}$ & $0.3 \mathrm{M}$ \\
Wide Field Optics & & \\
$40^{\prime} \mathrm{F} / 1-\mathrm{F} / 4$ & $1.8 \mathrm{M}$ & $1.3 \mathrm{M}$ \\
$60^{\prime} \mathrm{F} / 1.8-\mathrm{F} / 4.5$ & $4.0 \mathrm{M}$ & $3.0 \mathrm{M}$ \\
Aluminizing Tank, & $3.0 \mathrm{M}$ & $2.0 \mathrm{M}$ \\
Building \& Mirror Mover & & \\
MMT-Style Building & & \\
$7.5 \mathrm{~m} / 1$ & $4.2 \mathrm{M}$ & $2.8 \mathrm{M}$ \\
$7.5 \mathrm{~m} / 1.8$ & $11.8 \mathrm{M}$ & $7.9 \mathrm{M}$ \\
Mount and Drives & $5.1 \mathrm{M}$ & $3.4 \mathrm{M}$ \\
\hline
\end{tabular}

A first $F / 1$ telescope with both wide field and IR optics, plus an aluminizing facility would cost $\$ 20.5 \mathrm{M}$, with additional telescopes at that site, sharing the same coating facility costing $\$ 11.8 \mathrm{M}$, less if they are specialized for wide field or $I R$. If $F / 1$ could not be achieved, the corresponding estimates for an F/1.8 telescope are $\$ 30.3 \mathrm{M}$ for the first and $\$ 18.6 \mathrm{M}$ for repeat costs.

If several telescopes are to be used at the same site, then it is scientifically advantageous to mount them together as in the MMT. When phased and used with speckle or adaptive optics methods, the configuration of four telescopes we have developed as a straw man for the NNTT (Barr et al. 1983) gives 2.8 times the angular resolution, 8 times the areal resolution of the single mirrors. It is interesting then to try to compare costs, on the same 
basis, between four separately mounted and four co-mounted telescopes. Scaling the one-off cost by volume, we estimate the building cost to be $\$ 33.6 \mathrm{M}$ for a $4 \times 7.5 \mathrm{~m} \mathrm{~F} / 1.8 \mathrm{MMT}$. Little reduction is made going to $F / 1$ mirrors. Allowing $\$ 15.3 \mathrm{M}$ for the larger mount and drives, and $\$ 6 \mathrm{M}$ for the beam-combiner and rotating top end for quick configuration change, the MMT total is $\$ 88.8 \mathrm{M}$. This is more than our total of $\$ 56 \mathrm{M}$ for four $F / 1$ separately housed telescopes equipped for $40^{\prime}$ field and IR use, but about the same as for four $F / 1.8$ telescopes with $1^{\circ}$ field. Thus the MMT offers a strong scientific advantage at no additional cost compared to an array of $F / 1.8$ telescopes. It is more expensive than an array of four $F / 1$ telescopes, but offers the advantages of both high spatial resolution and wider field.

Our cost estimates are for Arizona, and for sites of elevation up to 10,700 ft. where working conditions are still comfortable. We estimate that construction and operations on an appreciably higher remote site would cost 308 more. Indeed if we increase our MMT cost estimate by this factor, they agree with Larry Barr's preliminary NNTT estimate. Clearly, if there are questions that affect the differential prices we suggest here, it is important that they should be sorted out. Equally it is important that the expected performance differences associated with these options should also be calculated in as much precision as we can muster, and their astronomical implications spelled out.

As a part of such an effort, we have tried to estimate what size unit telescope yields optimum $S / N$ per unit cost for observations at $10 \mu$, assuming a facillty in which the cost per unit increases as $D^{\mathbf{2 . 7}}$, and with the $r_{0}$ distribution suggested by Woolf and Ulich (1984). The optimization curve was rather flat, with a maximum somewhere in the vicinity of 7.5 to $10 \mathrm{~m}$. At longer wavelengths an optimum would be larger, and at shorter wavelengths the optimum would be smaller. On the other hand, for wide field multi-object studies in the visible, there is an initial cost for setting up the multi-object capability, atmospheric dispersion correction, etc. For such use the cost would need to be expressed as a set up cost plus $D^{2} .7$, plus a further term if detection is not noise free. For noise-free detection, the telescope cost is $A+D^{2.7}$, the cost per unit area is proportional to $A / D^{2}+D^{0.7}$, and the least cost per unit area is when the telescope cost is 2.857 times the cost of setting up for wide field observations. Since this cost includes spectrographs, multiple fiber feeds etc., it is clear that the appropriate telescope cost is at least several million dollars, and that the $7.5 \mathrm{~m}$ units cannot be far from optimum.

The one feature most noticably missing from our cost options is the ability to attain high angular resolution through long baseline. In previous papers, 
Low (1980), Woolf, Angel and McCarthy (1983) have suggested linear arrays for this purpose. The possibility of $F / 1$ telescopes is very advantageous for such systems in which the telescopes are moved so the baseline is always perpendicular to the object under observation. Fast primaries give smaller, lighter and stiffer units to be moved, with less need for enclosure protection. We envisage an interferometer with $7.5 \mathrm{~m}, \mathrm{~F} / 1$ mirrors on a $\sim 100 \mathrm{~m}$ baseline could be built on a structure like a rotating railway bridge. If two or more telescopes were separately mounted on such a bridge, either as a non redundant array, or as a Michelson interferometer, one could make pictures with an angular resolution as great as the size of the beam would admit. For such telescopes, MMT-type buildings would no longer be appropriate, but roll-off sheds could certalnly be built on the beam within the same budget.

A coating chamber on the bridge could be reached by a moving service enclosure with mirror handler. The cost of such a system with four mirrors would probably be comparable to that of the four telescope MMT. Lesser configurations such as the two mirror component of the versatile array (Woolf, Angel and McCarthy 1983; Bingham 1984) are more likely to be of possible consideration by us. However, there remains a lot of work to understand fully the scientific potential of the configurations.

Our aim in this section has been to create a do-it-yourself kit for costing a new observatory. Anyone can play the game, and the reader is invited to cost his or her favorite option. We have demonstrated here the range of options that are available with $7.5 \mathrm{~m}$ building blocks. We believe an important result of this study is that telescopes with fast $7.5 \mathrm{~m}$ mirrors should be in a price range accessible to major university astronomy departments, as well as to national or international observatories. It is our goal that the University of Arizona Observatories will construct one of the lesser facilities discussed in this paper, probably in the type of partnership we have already for the MMT and $10 \mathrm{~m}$ sub-millimeter telescopes.

\section{MOUNT GRAHAM}

Mount Graham, elevation $3267 \mathrm{~m},\left(32.7^{\circ} \mathrm{N}, 109.9^{\circ} \mathrm{W}\right)$ is a prime continental contender for the NNTT (Woolf and Merrill 1984). A map of Mt. Graham summit is shown in Figure 2. Mt. Graham's construction and operation costs from our existing experience are likely to be similar to those we have previously encountered, e.g.., at Mt. Hopkins.

Our search for a continental site was triggered by the discovery of the excellent seeing at the MMT, as good or better than that measured at any other 
terrestrial site (Woolf and Ulich 1984). It then appeared that the MMT seeing was about as good as could be expected from the measured atmospheric disturbances of the free atmosphere, with a modest site allowance.

An attempt to understand site contributions to seeing suggested to us that similar results to the MMT are likely to be available on a wide range of isolated mountains between $40^{\circ}$ and $20^{\circ}$ latitude. Estimates of poorer seeing are likely to have arisen from one of three factors (i) measures made too close to the ground, suffering excessively from the local ground inversion, (ii) facilities with standard poor thermal design in lower inland locations where temperature variations are relatively large, (iii) facilities in location where the seeing is disturbed by other nearby land of comparable height to windward. In regard to point (ii) we note that Dyck and Howell (1984) report that on Mauna $\mathrm{Kea}$, the temperature drop during the night is only $1.6 \pm 0.4^{\circ} \mathrm{C}$, mostly occurring during the first half. Under such circumstances, even thermally poor facilities using mirrors of high thermal inertia may perform quite well.

Our site selection was based on three principal criteria.

(1) Adequate height to permit IR and sub-mm observations into the edges of the far IR rotational absorption of $\mathrm{H}_{2} \mathrm{O}$.

(2) Minimum cloud cover.

(3) Freedom from artificial light sources for at least the next half century.

We believe item 2 is very important, and that the output of an observatory is a fairly sensitive function of the amount of clear sky (Evans 1969). Fortunately selection from the three criteria above seem to have yielded a mountain well isolated from others of comparable height to windward, and with two large summit areas relating somewhat differently to the prevailing wind.

Mt. Graham is about 150 road miles from Tucson. It has a paved road going most of the way to the summit, with the last few miles having a dirt road and reasonable grades. A program of comparison of this site with Mauna Kea has been started (Merrill and Forbes 1984). Initial studies of Mt. Graham prior to the comparison suggest that it is indeed likely to be competitive.

First, there are extensive studies of water vapor on Mt. Lemmon, which is in the same general airflow. Measures on Mt. Lemmon have been made with Westphal meters and placed on the same scale as similar measures with similar meters on Mauna Kea, e.g., Warner (1977). Such observations can only be made 
when the sky is clear, and the water vapor is not excessive by high. A number of similar comparisons also show that the water vapor on Mt. Graham is typically 0.8 of that on Mt. Lemmon. Secondly, there are radiosonde observations routinely made on the free atmosphere near both $\mathrm{Mt}$. Graham and Mauna Kea. These measures are very good when the air is moist, but unfortunately these balloon borne instruments cannot measure low values of relative humidity. One comparison of the two sites using both types of data has been made by Ulich (1984). Our independent study of the data suggests that the water vapor meter measures indicate a slight edge for $\mathrm{Mt}$. Graham for the ten months of the year excluding July and August, while the radiosonde data both from Gringorten et al. (1966), and a recent study by Wallace and Livingstone (1984) shows a slight edge to Mauna Kea over these same ten months. Our interpretation is that the range of water vapor variation is slightly greater in Arizona, as previously noted by Kuiper (1974) and that the instrument systematics discussed above produce the different results. Ulich infers that the annual amount of time for sub-mm observations, either those requiring extremely low water vapor, or those merely requiring less than $3 \mathrm{~mm}$ is the same at the two sites to within the precision of existing data. We should expect similar results at long $I R$ wavelengths.

We have been estimating the frequency of clear skies above Mt. Graham by making early morning photographs of the summit from the valley, and simultaneous observations of peaks nearer Tucson. These observations have shown a surprising absence of local cloud on Mt. Graham summit, indeed for most of the year it has had the clearest of the summits. However the limited data suggests also that the summer wet season lasts about seven days longer on Mt. Graham, and that this feature accounts for the increased cloudiness shown on cloud cover maps. The best comparison of overall clear skies ought to come from the long term Kitt Peak data as representative of Southern Arizona, and from the several years data now available from Mauna Kea. Unfortunately both sets of data are subjective, and are placed on a different basis. We are expecting that future long term studies will show a slight edge of clear skies to Mauna Kea, but this could well be wrong because of overinterpretation of poor data.

Winds are low on Mt. Graham, with simultaneous studies with Mt. Hopkins showing very similar wind levels at both sites. The wind at Mt. Hopkins is so low that we have in the past had studies of the MMT behaviour delayed considerably while we waited for high wind. One study of Mt. Hopkins wind is reported by Pearlman et al. (1971). 


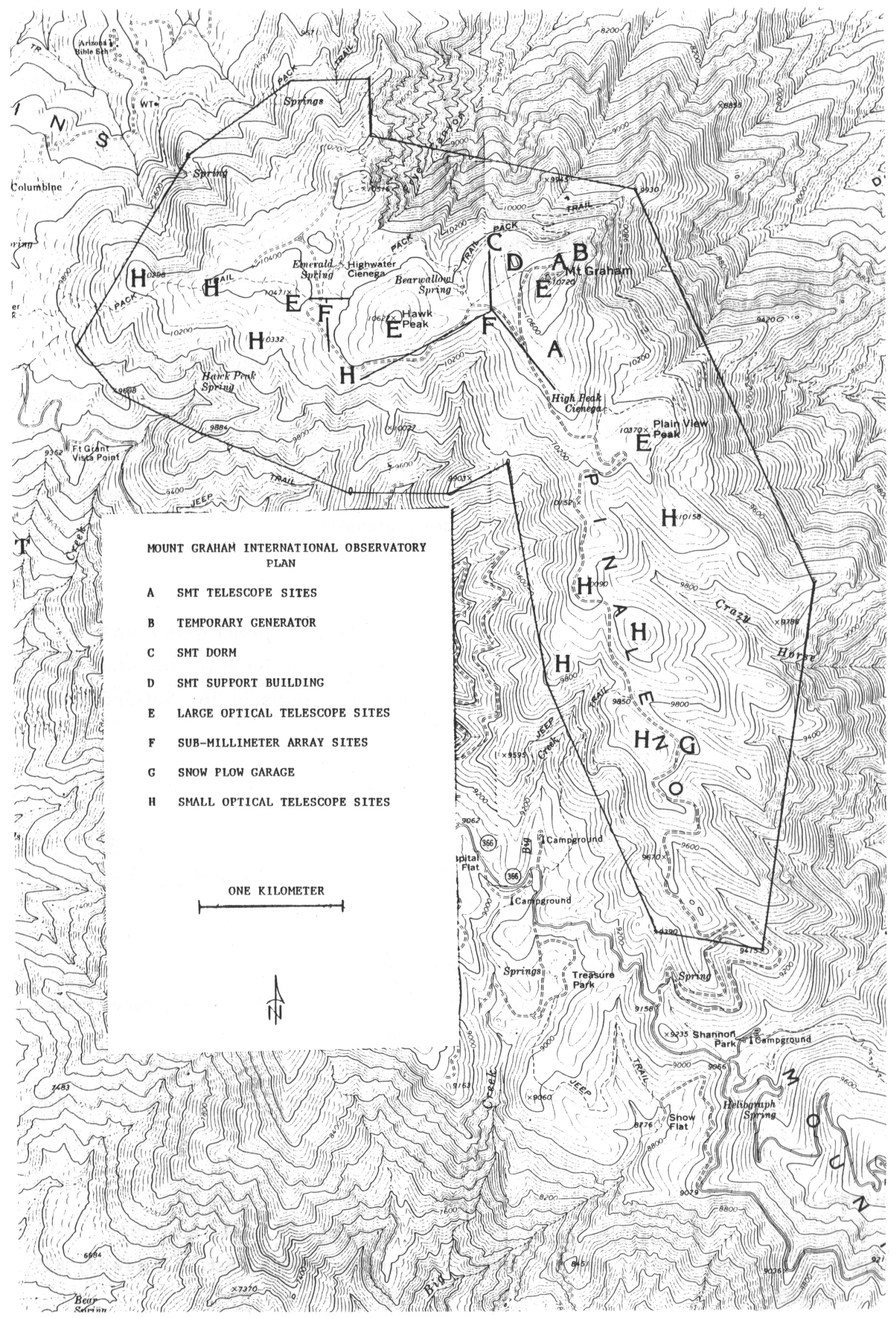


The least known aspect of Mt. Graham is its seeing. Modest size telescopes have been taken there several times, and have merely revealed diffraction disks. We do know that on the Heliograph Peak tower of the Forestry Service, a number of records of nighttime temperature variation show that for $90 \%$ of nighttime, variation is less than $0.5^{\circ} \mathrm{C}$ per hour, typical values being about half this. This variation appears to be similar to that reported for Mauna Kea by Dyck and Howell. This encourages us to believe that the seeing at appropriately selected locations on Mt. Graham will be dominated by the free atmosphere disturbances rather than by local effects, however we await the results of the NNTT program for clarification on this point.

Our plans are to open Mt. Graham summit as an International Observatory. The first telescope to go there will be the joint Max Planck Institute for Radioastronomie-University of Arizona $10 \mathrm{~m}$ Sub-mm Telescope. However, this mountain has many sites suitable for large optical-IR telescopes, and also fairly level areas that are appropriate for interferometry. We expect these features to prove important for further developments.

\section{ACKNOWLEDGEMENTS}

N. J. W. and J. R. P. A. would like to acknowledge support under NASA grant NACW-121. The work on Mt. Graham has been greatly assisted by the staff of the Fred L. Whipple Observatory, and by a number of groups in the Safford area.

\section{REFERENCES}

Angel, J. R. P., 1984, these proceedings.

Angel, J. R. P., Woolf, N. J., and Epps, H. W. 1982, Proc. SPIE, 332, 134.

Barr, L. D., Lynds, C. R., Angel, J. R. P., Woolf, N. J., Mast, T. S., and Nelson, J. E. 1983, Proc. SPIE, 444, 37.

Bingham, R. 1984, these proceedings.

Dyck, M. H., and Howell, R. R. 1983, PASP, 95, 786.

Epps, H. W., Angel, J. R. P., and Anderson, E. R. 1984, these proceedings.

Evans, D. S. 1969, Astron. Astrophys., 3, 247.

Gehrz, R. D., and Hackwell, J. E. 1978, Sky and Telescope, 55, 467.

Gringorten, I. I., Salmela, H. A., Solomon, I., and Sharp, J. 1966, Rept. AFCRL 66-621.

Hiltner, W. A. 1984, Verbal communication.

Humphries, C. M. 1984, these proceedings.

Kuiper, G. P. 1974, Comm. Lunar and Planetary Lab., 8, 121, and 337.

Low, F. J. 1980, Optical and IR Telescopes for the 1990's, Ed. A. Hewitt, 825, KPNO. 
Merrill, K. M., and Forbes, F. F. 1983, Proc. SPIE, 444, 148.

Pearlman, M. R., Hogan, D., Kirchhoff, W., Goodwin, K., Kurtenbach, D., Rocketto, S., and van't Sant B. 1971, Smithsonian Astrophysical Observatory Special Report \#327.

Robinson, L. J. 1982, Sky and Telescope, 64, 410 .

Ulich, B. 1984, Proposal to National Science Foundation for support for construction of sub-mm telescope facility.

Wallace, L., and Livingston, W. 1984, PASP, 96, 182.

Warner, J. 1977, PASP, 89, 724.

Woolf, N. J., Angel, J. R. P., and McCarthy, D. E. 1983, Proc. SPIE, 444.

Woolf, N. J., and Ulich, B. 1984, in ESO Workshop on Site Testing for Very Large Telescopes, ed. L. Woltjer and A. Andriesse.

Woolf, N. J., and Merrill, K. M. 1983, NNTT Technology Development Report \#4.

\section{DISCUSSION}

A. Labeyrie to R. Angel: This is a nice creative study. Do you believe there is a specific advantage in a linear array of 4 compared to a cross-shaped telescope? With a cross, the telescopes do not have to move faster, except if you go close to the horizon. It would be useful to further study and compare both array types.

N. Woolf: We felt that there is an advantage to having a relatively stable optical system, with the entire system moving rather than having separate telescopes with moving beam combiners. We too would like to join in a study which compares the options. 